\title{
Virtual reality applied to home-visit rehabilitation for hemiplegic shoulder pain in a stroke patient: a case report
}

\author{
Hiroki Funao', Mayumi Tsujikawa1', Ryo Momosaki², and Motomu Shimaoka ${ }^{3}$ \\ ${ }^{1}$ Course of Nursing Science, Graduate School of Medicine, Mie University, Japan \\ ${ }^{2}$ Department of Rehabilitation Medicine, Graduate School of Medicine, Mie University, Japan \\ ${ }^{3}$ Department of Molecular Pathobiology, Graduate School of Medicine, Mie University, Japan
}

\begin{abstract}
Objectives: Virtual reality (VR) has been shown to facilitate rehabilitation at hospitals by distracting patients' attention from pain and by providing a virtual environment favorable for motivating the patients to continue rehabilitation. However, the application of VR in a home-visit rehabilitation remains to be validated. Here, we report a case in which home-visit rehabilitation using immersive VR was effective for post-stroke hemiplegic shoulder pain.

Case presentation: After treatment, at a general hospital, for the hypertensive hemorrhage in the right brain capsule that resulted in the residual attention deficit disorder and left hemiplegia, a 63-year-old woman was cared for with a home-visit rehabilitation in a rural area. The patient had persistent pain in her left shoulder, which increased during activities of daily living and during rehabilitation, and the pain precluded rehabilitation. A VR relaxation program was delivered to the patient to alleviate pain during rehabilitation. Her shoulder pain was successfully alleviated using VR during training for muscle stretching and passive joint mobilization. Conclusion: The application of VR to home rehabilitation in rural areas may augment the effectiveness of home rehabilitation by alleviating pain during the procedure and sustaining the motivation for home rehabilitation.
\end{abstract}

Key words: home-visit rehabilitation, virtual reality, post-stroke hemiplegic shoulder pain

(J Rural Med 2021; 16(3): 174-178)

\section{Introduction}

Virtual reality (VR), which is a simulated pseudo-realistic experience, creates a virtual space and allows users to feel as if they were the in a real world. In recent years, VR has been applied to medical treatment to alleviate various types of pain, including acute pain during wound treatment, chronic pain lasting for more than three months, and cancer pain in terminally ill patients ${ }^{1,2)}$. The psychological effects of VR in inducing pain relief and enhancing motiva-

Received: January 19, 2021

Accepted: April 9, 2021

Correspondence: Hiroki Funao, Course of Nursing Science, Graduate School of Medicine, Mie University, 2-174 Edobashi, Tsu, Mie 514-8507, Japan

E-mail: h-funao@med.mie-u.ac.jp

This is an open-access article distributed under the terms of the Creative Commons Attribution Non-Commercial No Derivatives (by-nc-nd) License $<\mathrm{http}: / /$ creativecommons.org/ licenses/by-nc-nd/4.0/>. tion have been attracting attention as a means of promoting rehabilitation $^{3)}$. Several studies have reported the use of VR for the rehabilitation of post-stroke patients ${ }^{4-7)}$. Maintaining patients' positive attitudes toward rehabilitation, especially during repetitive training, is an important aspect of rehabilitation after stroke ${ }^{8)}$. This is especially the case with rehabilitation at home, in which patients are usually isolated, thereby having problems in keeping their motivation high for the treatment ${ }^{9,10)}$. Therefore, the psychological effects of VR in promoting motivation are expected to improve the effectiveness of home rehabilitation. However, applications of VR to home-based or domiciliary rehabilitation are limited, especially in rural areas. Here, we report the application of VR during home rehabilitation for a post-stroke patient living in a rural area in Japan who suffered hemiplegic shoulder pain that hindered effective rehabilitation.

\section{Case Presentation}

A 63-year-old woman living in a rural area developed a hypertensive hemorrhage on the right side of the inter- 
nal capsule and was rushed to the emergency room. The patient underwent craniotomy to remove the hematoma. The patient recovered well after the surgery. However, she developed left hemiparesis, dysphagia, left hemispatial neglect, and attention deficit disorder as sequelae of the hypertensive hemorrhage. The patient was then transferred to a rehabilitation hospital and underwent rehabilitation for three months. At the time of discharge, dysphagia and higher brain dysfunction had improved, but attention deficit disorder and left hemiparesis persisted. The patient also experienced pain in the left shoulder. In shoulder pain, considering the underlying medical conditions, such as a rotator cuff tear, is important in providing rehabilitation. According to the medical report, the pain was considered post-stroke hemiplegic shoulder pain, which is associated with a strong muscle tension, worsening of gliding ability, and myofascial adhesions. Therefore, after hospital discharge, home-visit rehabilitation was performed twice a week for $60 \mathrm{~min}$ for functional recovery and alleviation of left shoulder pain. Home-visit rehabilitation included muscle stretching, passive joint range of motion (ROM) training, load transfer training, activities of daily living (ADL) training, and gait training. The patient was on celecoxib medication for approximately 7 months before starting the VR rehabilitation. However, the pain persisted and increased during ADL and rehabilitation, which inhibited rehabilitation. Thus, to alleviate pain and motivate the patient, VR was used during home-visit rehabilitation procedures after obtaining written consent from the patient.

At the time of the VR application, in which the patient received home-visit rehabilitation service for one month, her ADL score was A2 (she rarely went out, and often slept during the day). The Barthel Index was 45 (10 for eating and transferring, 5 for dressing and toileting, 0 for bathing, walking, and climbing stairs, 5 for dressing, and 5 for controlling defecation and urination). The effect of cognitive function on the level of independence in daily life was ranked 1 . She suffered from mild dementia, which did not affect her daily activities or social life. The Brunnstrom recovery stage of her fingers and upper limbs was I, indicating no voluntary movement. Conversely, the Brunnstrom recovery stage of her lower limbs was III, indicating increased joint movement. The modified Ashworth scale (MAS) was used to measure spasticity of the elbow and knee. For the elbow, she had a MAS score of 2, indicating increased muscle tone throughout the entire ROM but well-maintained muscle tone during other movements. For the knee, she had a MAS score of +1 , indicating a slight increase in muscle tone, manifested by a catch, followed by continued resistance throughout the remaining ROM.

An immersive VR-based relaxation program was used during muscle stretching and passive joint mobilization training to alleviate her shoulder pain (Figure 1). The Applied VR (AVR) healthcare platform (AppliedVR Inc., Los Angeles, CA, USA) was used in this case, which provides an array of analgesic programs. Among more than 20 different VR programs for distraction and relaxation, "Dream Beach" and "Wild Dolphins" were selected according to the patient's preferences; the former provided the experience of being on a calm beach on a sunny day, while the latter provided the experience of swimming with wild dolphins (Figure 2). We set up and installed a head-mounted display (HMD) and headphones required for the VR use. The patient reported no episodes of anxiety regarding the use of VR. Each program lasted for $30 \mathrm{~min}$. The patient continued to take medications during the VR rehabilitation.

The degree of left shoulder pain during the training for muscle stretching and passive ROM is shown in Figure 3 (left shoulder pain during muscle stretching and passive joint mobilization training). The degree of left shoulder pain was measured before, during, and after rehabilitation using the Numeric Rating Scale (NRS) score for pain $(0-10)^{11)}$. Before the application of VR, the left shoulder pain during rehabilitation was 10 points. After five attempts of VR-as-

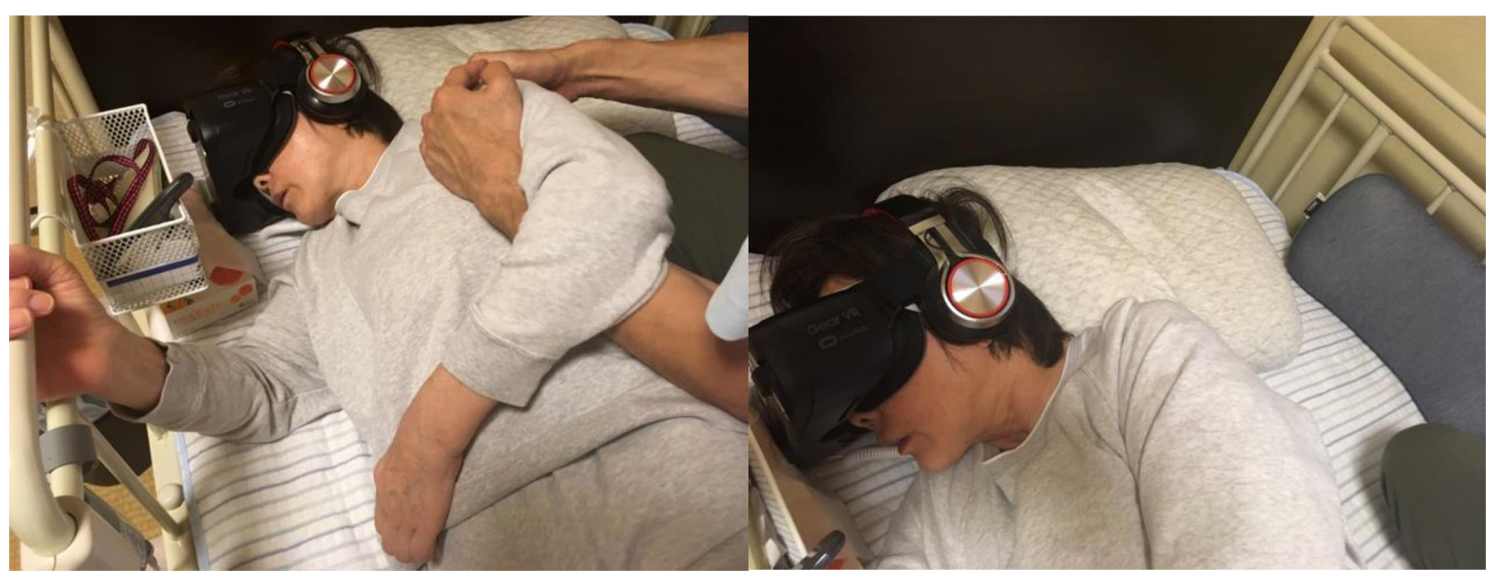

Figure 1 Virtual reality (VR)-based joint mobilization and passive joint range-of-motion training. 


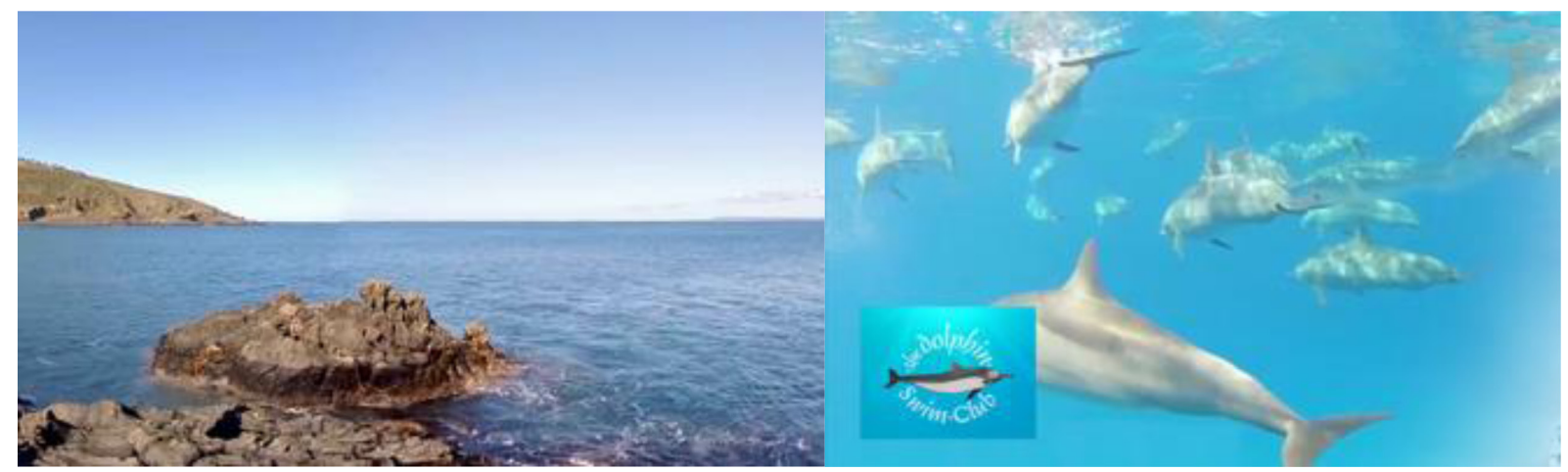

Figure 2 The actual VR images (Picture on the left: "Dream Beach", Picture on the right: "Wild Dolphins").

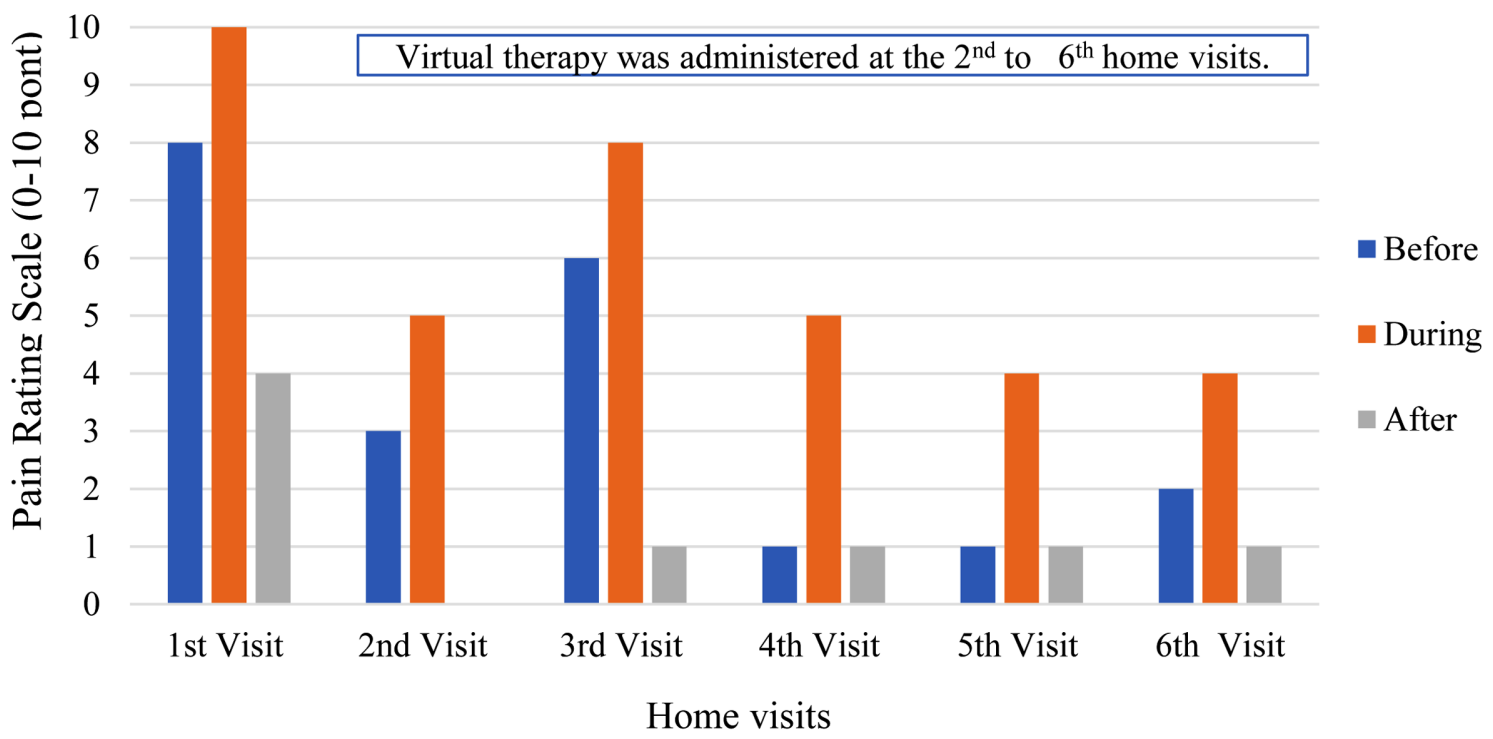

Figure 3 Left shoulder pain during muscle stretching and passive joint mobilization training.

sisted home-visit rehabilitation over 3 weeks, there were few changes in the functions of the right upper limb or ADL. However, the patient showed a decrease in the left shoulder pain from 10 points to $4-8$ points during rehabilitation and from 4 points to $0-1$ points after rehabilitation. This suggested a reduction in pain, and the visiting physical therapist reported a decrease in left shoulder muscle tone and an increase in joint ROM (measurements were not taken) at the third to fifth visits.

\section{Discussion}

Stroke is prevalent in elderly people, causing major disability that often compels patients to require assistance with daily living activities ${ }^{7}$. Rehabilitation after stroke is extremely important; however, hemiplegic shoulder pain is a common complication after stroke that often hinders rehabilitation $^{12}$. Failure to deliver effective rehabilitation could result in a decreased shoulder ROM and reduced functional use of the arm, thereby limiting patients' performance and participation in rehabilitation. Hemiplegic shoulder pain consequently delays their discharge from medical institutions $^{12,13)}$ and reduces the quality of life, which has a negative impact on mood ${ }^{14-16)}$. Many treatment modalities, including physical therapy, massage therapy, shoulder positioning, local injections, and oral medications, have been applied to normalize muscle tone and increase $\mathrm{ROM}^{17}$; however, these conventional therapies have not been shown to relieve refractory hemiplegic shoulder pain ${ }^{18)}$.

The VR program used in this case alleviated pain by distracting the patient's attention from pain. The alleviation of pain is thought to be the psychological effect of immersion in the virtual space created by the VR technology $y^{1}$. Another mechanism by which the VR program reduced pain in this case would be the creation of a relaxing atmosphere. Perception of pain is influenced by the patient's affect ${ }^{19)}$. Therefore, the positive effect induced by the psychological effects of the VR relaxation program could suppress pain perception. The 
effects of VR to induce both distraction and relaxation would synergistically relieve pain perception in patients, thereby promoting rehabilitation. Pain scores were the highest during rehabilitation in all sessions. However, pain scores during rehabilitation decreased from a maximum of 10 to 4 . Since the rate of change used to define a positive outcome for pain as expressed by the NRS is $\geq 33 \%{ }^{20)}$, we consider the rate of change in this study $(60 \%)$ to be a significant change. During the course of receiving multiple VR rehabilitation sessions, the basal levels of pain gradually decreased before, during, and after the rehabilitation procedures, thereby suggesting the effectiveness of the VR rehabilitation.

Approximately $40 \%$ of home-visit rehabilitation users in Japan require stroke rehabilitation. The case reported here is a typical example of home-visit rehabilitation users in Japan. Home rehabilitation services by specialized medical professionals, such as physical therapists and occupational therapists, are provided to patients who have difficulty visiting rehabilitation facilities, such as medical institutions, or do not have sufficient physical capabilities to visit hospitals. In contrast to rehabilitation at hospitals, rehabilitation at home usually lacks important resources to implement sustainable and effective treatment. One such resource is the environment to maintain patients' positive attitude toward rehabilitation, especially during repetitive training, which is an important aspect of rehabilitation after stroke ${ }^{21)}$. The lack of resources necessary for sustainable and effective rehabilitation at home is even more significant in rural areas of Japan that confront the rapidly aging population and low birthrate ${ }^{9}$. Moreover, the number of home visits per day is limited due to the low population density in those areas, which can easily become unprofitable ${ }^{9}$. Therefore, a virtual space created by the VR technology may provide an alternative to the supportive environment that maintains patients' positive attitudes, thereby improving the effectiveness of rehabilitation $^{5,6}$. The results of this study suggest that VR may help augment the effect of home rehabilitation in rural areas.

The VR programs used in this study were designed to realize a virtual experience at the beach and ocean. From our preliminary results of VR-assisted home-visit rehabilitation for several patients, we learned that they favored the virtual experience at the beach and the ocean, as they immensely missed the sea but their disabilities prevented them from going out. Home-visit rehabilitation in rural areas in Japan often lacks resources to support outing activities for patients with disabilities. Therefore, VR may be able to create a positive psychological change in patients by providing them outing experience and maintaining their motivation to perform repetitive training at home, which is difficult to continue, and further, improve their quality of life.

Although immersion in the virtual space created by the VR technology is important to alleviate pain ${ }^{1)}$, to immerse oneself in the virtual space, spatial cognitive function must be maintained. In this case, after developing left hemispatial neglect, the patient's spatial cognitive function recovered to a sufficient degree to use the VR program, which allowed her to immerse in the virtual space. Most studies that used VR in stroke patients listed spatial cognitive function as the exclusion criteria ${ }^{22)}$; it must be fully considered that the patient's spatial cognitive functions are preserved. In addition, the clinical effectiveness has yet to be established, and a few reports have described the application of VR in the acute phase of rehabilitation to improve upper limb muscle strength, functional status, and mood states such as depression and anxiety ${ }^{23-25)}$.

Although VR is generally considered to be a safe and non-invasive procedure with few side effects, the use of VR has been reported to induce VR sickness, such as nausea and headachel), which the present case did not suffer. One must be aware of the potential occurrence of VR sickness and carefully observe the patient's condition during the administration of VR. An obstacle to introducing VR to home-visit rehabilitation in rural areas in Japan is the fact that more than $70 \%$ of patients are aged $\geq 75$ years $^{26)}$. The successful application of VR to senior individuals may require a careful introductory session.

The patient provided informed consent for the publication of this case. The study was performed in accordance with the Declaration of Helsinki and ethical guidelines for medical and health research involving human subjects in Japan.

Conflict of interest: The authors declare no conflicts of interest associated with this manuscript.

\section{References}

1. Ahmadpour N, Randall H, Choksi H, et al. Virtual Reality interventions for acute and chronic pain management. Int J Biochem Cell Biol 2019; 114: 105568. [Medline] [CrossRef]

2. Niki K, Okamoto Y, Maeda I, et al. A novel palliative care approach using virtual reality for improving various symptoms of terminal cancer patients: A preliminary prospective, multicenter study. J Palliat Med 2019; 22: 702-707. [Medline] [CrossRef]

3. Rose T, Nam CS, Chen KB. Immersion of virtual reality for rehabilitation—review. Appl Ergon 2018; 69: 153-161. [Medline] [CrossRef]

4. Kilbride C, Scott DJM, Butcher T, et al. Rehabilitation via Home Based gaming exercise for the Upper-limb post Stroke (RHOMBUS): protocol of an 
intervention feasibility trial. BMJ Open 2018; 8: e026620. [Medline] [CrossRef]

5. Valdés BA, Glegg SMN, Lambert-Shirzad N, et al. Application of commercial games for home-based rehabilitation for people with hemiparesis: challenges and lessons learned. Games Health J 2018; 7: 197-207. [Medline] [CrossRef]

6. Standen PJ, Threapleton K, Richardson A, et al. A low cost virtual reality system for home based rehabilitation of the arm following stroke: a randomised controlled feasibility trial. Clin Rehabil 2017; 31: 340-350. [Medline] [CrossRef]

7. Standen PJ, Threapleton K, Connell L, et al. Patients' use of a home-based virtual reality system to provide rehabilitation of the upper limb following stroke. Phys Ther 2015; 95: 350-359. [Medline] [CrossRef]

8. Chanques G, Viel E, Constantin JM, et al. The measurement of pain in intensive care unit: comparison of 5 self-report intensity scales. Pain 2010; 151: 711-721. [Medline] [CrossRef]

9. Jurkiewicz MT, Marzolini S, Oh P. Adherence to a home-based exercise program for individuals after stroke. Top Stroke Rehabil 2011; 18: 277-284 [Medline] [CrossRef]

10. Barker RN, Brauer SG. Upper limb recovery after stroke: the stroke survivors' perspective. Disabil Rehabil 2005; 27: 1213-1223. [Medline] [CrossRef]

11. Jeon WH, Park GW, Jeong HJ, et al. The comparison of effects of suprascapular nerve block, intra-articular steroid injection, and a combination therapy on hemiplegic shoulder pain: pilot study. Ann Rehabil Med 2014; 38: 167-173. [Medline] [CrossRef]

12. Roth EJ. Trends in stroke rehabilitation. Eur J Phys Rehabil Med 2009; 45: 247-254. [Medline]

13. Griffin JW. Hemiplegic shoulder pain. Phys Ther 1986; 66: 1884-1893. [Medline] [CrossRef]

14. Huang YC, Chang KH, Liou TH, et al. Effects of Kinesio taping for stroke patients with hemiplegic shoulder pain: a double-blind, randomized, placebocontrolled study. J Rehabil Med 2017; 49: 208-215. [Medline] [CrossRef]

15. Van Til JA, Renzenbrink GJ, Groothuis K, et al. A preliminary economic evaluation of percutaneous neuromuscular electrical stimulation in the treatment of hemiplegic shoulder pain. Disabil Rehabil 2006; 28: 645-651. [Medline] [CrossRef]

16. Adey-Wakeling Z, Liu E, Crotty M, et al. Hemiplegic shoulder pain reduces quality of life after acute stroke: a prospective population-based study. Am J Phys Med Rehabil 2016; 95: 758-763. [Medline] [CrossRef]

17. Lindgren I, Gard G, Brogårdh C. Shoulder pain after stroke - experiences, consequences in daily life and effects of interventions: a qualitative study. Disabil Rehabil 2018; 40: 1176-1182. [Medline] [CrossRef]

18. Kasapoğlu-Aksoy M, Aykurt-Karlıbel I, Altan L. Comparison of the efficacy of intramuscular botulinum toxin type-A injection into the pectoralis major and the teres major muscles and suprascapular nerve block for hemiplegic shoulder pain: a prospective, double-blind, randomized, controlled trial. Neurol Sci 2020; 41: 2225-2230. [Medline] [CrossRef]

19. Finan PH, Garland EL. The role of positive affect in pain and its treatment. Clin J Pain 2015; 31: 177-187. [Medline] [CrossRef]

20. Farrar JT, Berlin JA, Strom BL. Clinically important changes in acute pain outcome measures: a validation study. J Pain Symptom Manage 2003; 25: 406-411. [Medline] [CrossRef]

21. Sencan S, Celenlioglu AE, Karadag-Saygı E, et al. Effects of fluoroscopy-guided intraartıcular injection, suprascapular nerve block, and combınation therapy in hemiplegic shoulder pain: a prospective double-blınd, randomized clınical study. Neurol Sci 2019; 40: 939-946. [Medline] [CrossRef]

22. Laver KE, Lange B, George S, et al. Virtual reality for stroke rehabilitation. Cochrane Database Syst Rev 2017; 11: CD008349. [Medline]

23. Kwon JS, Park MJ, Yoon IJ, et al. Effects of virtual reality on upper extremity function and activities of daily living performance in acute stroke: a doubleblind randomized clinical trial. NeuroRehabilitation 2012; 31:379-385. [Medline] [CrossRef]

24. Patel J, Fluet G, Qiu Q, et al. Intensive virtual reality and robotic based upper limb training compared to usual care, and associated cortical reorganization, in the acute and early sub-acute periods post-stroke: a feasibility study. J Neuroeng Rehabil 2019; 16: 92. [Medline] [CrossRef]

25. Lin RC, Chiang SL, Heitkemper MM, et al. Effectiveness of early rehabilitation combined with virtual reality training on muscle strength, mood state, and functional status in patients with acute stroke: a randomized controlled trial. Worldviews Evid Based Nurs 2020; 17: 158-167. [Medline] [CrossRef]

26. Ministry of Health, Labor and Welfare. Surveys related to verification of the effects of the revision of nursing care fees and survey research. 2015. (in Japanese) 